\title{
Status of the connection between unidentified EGRET sources and supernova remnants: The case of CTA 1
}

\author{
Diego F. Torres ${ }^{1}$, Thomas M. Dame ${ }^{2}$ \& Gustavo E. Romero ${ }^{3}$ \\ ${ }^{1}$ Lawrence Livermore Laboratory, 7000 East Ave. L-413, Livermore, CA 94550, \\ $U S A$ \\ ${ }^{2}$ Harvard-Smithsonian Center for Astrophysics, 60 Garden Street, Cambridge, MA \\ 02138, USA \\ ${ }^{3}$ Instituto Argentino de Radioastronomía, C.C.5, 1894 Villa Elisa, Buenos Aires, \\ Argentina
}

\begin{abstract}
In this paper we briefly comment on the observational status of the possible physical association between unidentified EGRET sources and supernova remnants (SNRs) in our Galaxy. We draw upon recent results presented in the review by Torres et al. (Physics Reports, 2003), concerning molecular gas in the vicinity of all 19 SNRs found to be positionally coincident with EGRET sources at low Galactic latitudes. In addition, we present new results regarding the supernova remnant CTA 1. Our findings disfavor the possibility of a physical connection with the nearby (in projection) EGRET source. There remains possible, however, that the compact object produced in the supernova explosion be related with the observed $\gamma$-ray flux.
\end{abstract}

Keywords: Supernova remnants - gamma rays: observations - interstellar medium

\section{A brief overview of the EGRET-SNR connection}

We have recently presented a case-by-case analysis of the positional correlation between unidentified EGRET sources and supernova remnants (SNRs, Torres et al. 2003). There are 19 EGRET sources at low Galactic latitudes $\left(|b|<10^{\circ}\right)$ in spatial correlation with, mostly shelltype, SNRs. The Poisson probability for the 19 coincidences to be a chance effect is $1.05 \times 10^{-5}$, i.e., there is an a priori 0.99998 probability that at least one of the positional associations is physical. This expected chance association was computed using a numerical code described by Romero et al. (1999), and Sigl et al. (2001). With the aim to identify the most plausible candidates for an EGRET-SNR connection, we have analyzed, among other things, the variability status of the EGRET sources and the potential contribution from known radio pulsars to the high energy flux (Torres et al. 2003).

In order to understand the origin of all the unidentified detections, their variability status is of paramount importance (Nolan 2003, Torres et al. 2001). Classic known models for $\gamma$-ray sources in our Galaxy, like a SNR-molecular cloud interaction (e.g. Aharonian \& Atoyan 1996),

(C) 2017 Kluwer Academic Publishers. Printed in the Netherlands. 
would produce non-variable detections during the timescale of EGRET observations. To classify the variability status we take into account both indices quoted in the literature, Torres et al.'s (2001) I and Tompkins' $\tau$. They are different in nature, although results have proven to be statistically correlated (Torres, Pessah \& Romero 2001). The index $I$ is a relative classification of variability with respect to the pulsar population. Sources with $I>2.5$ are more than $3 \sigma$ away from the statistical variability of pulsars. Similarly, sources for which $\tau$ is at least 0.6 , are also more than $3 \sigma$ away from the mean value of the $\tau$ upper limit for pulsars, and are thus classified as variable. For comparison, note that the mean value of $\tau(I)$ for known AGNs is 0.9 (3.3). We find that most EGRET sources in positional coincidence with SNRs, and certainly those which represent the best cases for a physical association, are non-variable.

In order to unambiguously identify a pulsar as the origin of the $\gamma$ rays from an EGRET source, $\gamma$-ray pulsations must be detected at the pulsar period. However the $\gamma$-fluxes are generally very low, and nearly contemporaneous radio/X-ray and $\gamma$-ray observations are required to fold the few available photons with the correct ephemeris. Because this is no longer possible owing to the demise of the Compton GammaRay Observatory, new candidate associations must mostly be judged by comparison with the properties of the six known EGRET pulsars and those of the known best candidates. We have done so for every known radio pulsar found to be coincident with these 19 EGRET sources, and have isolated several interesting candidates for an EGRET source-pulsar connection. These are mostly found with young pulsars pertaining to the recently released Parkes Multibeam Survey (see, e.g. Torres, Butt, \& Camilo 2001, Torres \& Nuza 2003). This scenario, then, is a competing one to the EGRET-SNR connection and has to be taken into account in judging the plausibility of the latter.

The need of a case-by-case analysis is then clearly established. Several cases for a physical association, based on the analysis of $\gamma$-ray data, the molecular environment of the SNR, and the analysis of any coincident pulsar (if any), have been isolated and reviewed in Torres et al. (2003). Among the most notable cases for such a physical association are G347.3-0.5, W66, W28 and IC443. The first of these, providing probably the best evidence to date for a hadronic origin of the $\gamma$-ray source detected by EGRET, has received much attention recently (Butt et al. 2001, 2002, Enomoto 2002, Uchiyama et al. 2002). In addition, the case-by-case analysis shows that it is at least plausible, contrary to expectations, that EGRET has detected distant (more than $6 \mathrm{kpc}$ ) SNRs. There are 5 coinciding pairs of 3EG sources and SNRs for which the latter apparently lie at such high values of distance (disregarding 
those related with SNRs spatially close to the galactic center). For all these cases, we have uncovered the existence of nearby, large, in some cases giant, molecular clouds that could enhance the GeV signal through pion decay. It is possible that the physical relationship between the 3EG source and the coincident SNR could provide for these pairs a substantial part of the $\mathrm{GeV}$ emission observed. This does not preclude, however, composite origins for the total amount of the radiation detected, since some of these cases present other plausible scenarios (e.g. energetic pulsars in the EGRET field). AGILE observations, in advance of GLAST, would greatly elucidate the origin for these 3EG sources, since even a factor of 2 of improvement in spatial resolution would be enough to reject the EGRET-SNR connection.

In what follows we attempt to briefly analyze, similarly to what was done in the case-by-case analysis of Torres et al. (2003), the pair CTA 1/3EG J0010+7309, a supernova remnant lying at relatively high Galactic latitudes, and for which there has been little analysis on their $\mathrm{CO}$ environment.

\section{CTA 1: General Features}

CTA 1 is a shell-type SNR, but its shell is incomplete and brokenout towards the NW. This breakout phenomenon may be caused by more rapid expansion of the blast wave shock into a lower density region toward the NW. HI observations supported this interpretation (Pineault et al. 1993, 1997). The latter papers place the SNR at $1.4 \pm 0.3$ $\mathrm{kpc}$, the kinematic distance corresponding to the systemic velocity of a partial HI shell $(-16 \mathrm{~km} / \mathrm{s})$ that the authors find to the NW. However, it is to be noticed that the systemic (unperturbed) velocity of a partially expanding shell might be subject to a great uncertainty.

Since CTA 1 is located at a relatively high latitude $\left(b=10^{\circ}\right)$ and it is nearby, it has a large angular size (90 arcmin), little foreground or background confusion, and it can be observed at exceptionally high linear resolution. The age of the SNR was estimated to be $10^{4} \mathrm{yr}$ by Pineault et al. (1993), but it could be younger by a factor of 2 (Slane et al. 1997). CTA 1 was subject of intense observational campaigns in the past years. There have been both ROSAT and ASCA X-ray observations (Seward et al. 1995, Slane et al. 1997), as well as optical, infrared and radio studies (see Pineault et al. 1997 and Brazier et al. 1998 for a review).

CTA 1 belongs to the class of composite SNRs (SNRs with central pulsar wind nebulae), which show a shell-type morphology in the radio band and are center-filled in X-rays. Five point sources were detected 
with ROSAT, one of which was found to coincide with the EGRET source (at the time of the analysis, 2EG J0008+7307), see Figure 3 of Brazier et al. (1998). ASCA data suggested that this source, named RX J0007.0+7302, has a non-thermal spectrum, and gave support to the idea that it is the pulsar left from the supernova explosion (Slane et al. 1997). Recent observations by Slane et al. (2004), using XMM, confirm that the X-ray spectrum of the source is consistent with that of a neutron star and is well described by a power law with the addition of a soft thermal component that may correspond to emission from hot polar cap regions or to cooling emission from a light-element atmosphere over the entire star. An extrapolation of the non-thermal spectrum of RX J0007.0+7302 to $\gamma$-ray energies would yield a flux consistent with that of EGRET source 3EG J0010+7309, supporting the proposition that these sources could be related. A Chandra image of the central X-ray source RX J0007.0+7303 (Halpern et al. 2004) reveals a point source, a compact nebula, and a bent jet, all of which are characteristic of energetic, rotation-powered pulsars.

Optical observations were carried out by Brazier et al. (1998), with a 2.12-m telescope, but no object was found within the positional error box of the X-ray source. This allowed an upper limit to be set on the optical magnitude of any counterpart to the putative pulsar. Halpern et al. (2004) also obtained upper limits in the optical at the position of the point source, corresponding to an X-ray-to-optical flux ratio larger than 100. Neither a VLA image at $1425 \mathrm{MHz}$ nor a deep pulsar search at $820 \mathrm{MHz}$ using the NRAO Green Bank Telescope, revealed a radio pulsar counterpart

The $\gamma$-ray source 3EG J0010+7309 is non-variable under the $I$ and $\tau$ schemes, and has a hard spectral index of $1.85 \pm 0.10$, compatible with those of the Vela pulsar. This source was also detected in the Second EGRET Catalog, but with a shifted position which made it coincide with a nearby AGN - which was at the time proposed as a possible counterpart (Nolan et al. 1996). This AGN is not coincident with the 3EG source and can not be considered a plausible counterpart any longer. Based on positional coincidence, on the hard spectral index, and on physical similarities between the Vela pulsar and RX J0007.0+7302, Brazier et al. (1998) proposed that the 3EG source and this X-ray source were related. For an assumed 1 sr beaming, the observed 100-2000 MeV flux corresponds to a luminosity of $4 \times 10^{33}$ erg $\mathrm{s}^{-1}$, compatible with other $\gamma$-ray pulsar detections. A competing explanation to the $\gamma$-ray pulsar would be given if a massive and dense molecular cloud is found to be interacting with, or being overtaken by, the supernova remnant shock. The molecular environment of CTA 1 is analyzed below to further assess this possibility. 
Figure 1. Molecular gas in the direction of the SNR CTA 1. (a) The false-color map is $\mathrm{CO}$ integrated over the velocity range -15 to $+5 \mathrm{~km} / \mathrm{s}$. The black contours are 4.85 GHz intensity from the survey of Condon et al. (1994), which show CTA 1 as a semi-circular feature near the center of the map. The red (lighter) contours are the $50 \%, 68 \%, 95 \%$, and $99 \%$ confidence contours for the unidentified gamma ray source 3EG J0010+7309. (b) A CO latitude-velocity map integrated from $116^{\circ}$ to $120^{\circ}$ in Galactic longitude. [[The figure is available on-line as a jpeg file]]

As can be noted from the figure, the EGRET source seems too far from the SNR nearest rim. However, if some SNRs interact, as expected, with nearby massive clouds producing enhanced $\gamma$-ray emission through hadronic/Bremsstrahlung interactions, cases in which there is just a marginal coincidence between the SNRs and the centers of the EGRET sources should be also considered, since the peak $\gamma$-ray emissivity will likely be biased towards the adjacent cloud.

\section{CTA 1: Molecular environment}

As discussed in Dame et al. (1987), much of the local (closer than Perseus Arm) CO emission in the second quadrant of the Galaxy bifurcates into 2 velocity components, one centered near $-12 \mathrm{~km} / \mathrm{s}$ and the other close to $0 \mathrm{~km} / \mathrm{s}$. Dame et al. (1987) argue that the "-12 $\mathrm{km} / \mathrm{s}$ clouds" are probably associated with a string of OB associations at 800 to $1000 \mathrm{pc}$ from Earth. The clouds with velocities near $0 \mathrm{~km} / \mathrm{s}$ were associated with the so-called "Lindblad Ring" at 300 pc. The long thin cloud partially overlapping CTA 1 (see Figure 1) has a mean velocity of $\sim-3 \mathrm{~km} / \mathrm{s}$, suggesting that it is part of the Lindblad Ring population. In fact, these clouds appear to be part of a very large loop of local clouds (all in the range -5 to $0 \mathrm{~km} / \mathrm{s}$ ). Grenier et al. (1989) proposed that this loop surrounds a $4 \times 10^{4}$ yr old Type I SNR $300 \mathrm{pc}$ away. Thus, the velocity of the cloud near to CTA 1 suggests that it is part of this larger system, lying at only $\sim 300 \mathrm{pc}$.

The total $\mathrm{H}_{2}$ mass (calculated from $\mathrm{CO}$ data) of the long thin cloud near CTA1 (in the region $l=116^{\circ}$ to $120^{\circ}, b=8^{\circ}$ to $13^{\circ}, v=-15$ to $5 \mathrm{~km} / \mathrm{s}$ ) is $4900 M_{\odot}$, assuming a distance of $300 \mathrm{pc}$. However, there is no evidence of interaction between the remnant and the cloud (what is consistent with the different most plausible distances to each of these objects). Figure $1 \mathrm{~b}$ attached, for example, shows a latitude-velocity

map integrated over the longitude extent of the cloud. There is little indication of accelerated gas at the latitude/velocity of the remnant, $b=9^{\circ}$ to $10^{\circ}$ and $\sim-15 \mathrm{~km} / \mathrm{s}$, respectively. The cloud may well be, then, in the foreground, and unrelated to CTA 1. In addition to this, 
there is no $\mathrm{CO}$ emission detected within the contours of the 3EG source, which also argues against a hadronic origin of the $\gamma$-ray source.

Pineault et al.'s (1993) systemic velocity of $-16 \mathrm{~km} / \mathrm{s}$ for the HI shell is reasonable close to that of the " $-12 \mathrm{~km} / \mathrm{s}$ clouds", which in turn are probably associated with the OB associations Cep OB2, Cep OB3, Cep OB4, and Per OB3 at $\sim 800$ pc. This might be suggesting that the latter is the most likely distance for CTA 1, although it remains uncertain. In any case, it seems that CTA 1 and 3EG J0010+7309 might be physically related only through the compact object left by the supernova explosion, RX J0007.0+7302. A better localization of the $\gamma$ ray source with AGILE and GLAST will certainly test this suggestion. For the moment, our $\mathrm{CO}$ analysis shows that a molecular cloud origin of the $\gamma$-ray source 3EG J0010+7309 (for instance, through hadronic processes similar to the case of G347.3-0.5 proposed by Butt et al. 2001) is untenable.

\section{Acknowledgments}

The work of DFT was performed under the auspices of the U.S. Department of Energy (NNSA) by University of California Lawrence Livermore National Laboratory under contract No. W-7405-Eng-48. G.E.R. was supported by CONICET (under grant PIP $\mathrm{N}^{\circ}$ 0430/98), ANPCT (PICT 03-04881), as well as by Fundación Antorchas. We thank P. Slane for comments.

\section{References}

Brazier K.T.S., Reimer O., Kanbach G., \& Carramiñana A. 1998, MNRAS 295, 819 Butt Y., et al. 2001, ApJ 562, L167

Butt Y., et al. 2002, Nature 418, 499

Condon J.J., et al. 1994, AJ 107, 1829

Dame T.M., et al. 1987, ApJ 322, 706

Enomoto R., et al. 2002, Nature 416, 823

Grenier I. et al. 1989, ApJ 347, 231

Halpern J. P., Gotthelf E. V., Camilo F., Helfand D. J. \& Ransom S. M. 2004, astro-ph/0404312

Nolan P.L., et al. 1996, ApJ 459, 100

Nolan P.L., Tompkins W.F., Grenier I.A., \& Michelson P.F. 2003, ApJ 597, 615

Pineault S., et al. 1993, AJ 105, 1060

Pineault S., et al. 1997, A\&A 324, 1152

Slane P., Zimmerman E. R., Hughes J. P., Seward F. D., Gaensler B. M. \& Clarke M. J. 2004, ApJ 601, 1045

Romero G.E., Benaglia P., \& Torres D.F. 1999, A\&A 348, 868

Seward F.D., Schmidt B., \& Slane P. 1995, ApJ 453, 284 
Sigl G., et al. 2001, Phys. Rev. D63, 081302

Slane P., et al. 1997, ApJ 485, 221

Torres D.F., et al. 2001, A\&A 370, 468

Torres D.F., Pessah M.E., \& Romero G.E. 2001c, Astronomische Nach. 322, 223

Torres D.F., Butt Y.M. \& Camilo F. 2001, ApJ 560, L155

Torres D.F., \& Nuza S. E. 2003, ApJ 583, L25

Torres D.F., et al. 2003, Physics Reports 382, 303

Uchiyama Y., Takahashi T., \& Aharonian F.A. 2002a, PASJ54, L73 
snr-cta1-web.tex; 2/07/2017; $1: 15 ;$ p.8 
This figure "cta1.jpg" is available in "jpg" format from: http://arxiv.org/ps/astro-ph/0406335v2 